\title{
A study on the differential and sub-differential of fuzzy mapping and its application problem
}

\author{
Yu-E Bao*, Jin-Jun Li \\ College of Mathematics, Inner Mongolia University for Nationalities, Inner Mongolia, Tongliao City, 028043, China.
}

Communicated by S. K. Samanta

\begin{abstract}
In this paper, firstly, we gain some basic properties and characterization theorems of the differential and local sub-differential of the fuzzy mapping, obtain an important result that the local sub-differential of fuzzy mapping is an empty set or a convex set. Secondly, we generalize the concept of local differentiability of fuzzy mapping, and obtain some basic properties about the concept. At last, we study the relationships between sub-differential of fuzzy mapping and differential of convex fuzzy mappings. Moreover, a sufficient condition that a class of fuzzy mapping have convex extension is gained. (C)2017 all rights reserved.
\end{abstract}

Keywords: Fuzzy number, fuzzy mapping, differential (sub-differential), convexification fuzzy mapping, convex extension. 2010 MSC: 03E72, 52A41, 58C20, 58C25.

\section{Introduction}

In 1972, Chang and Zadeh introduced the concept of fuzzy number in paper [6], from then on, many scholars conducted in-depth research for fuzzy mapping (fuzzy value function in fuzzy number), and obtained a series of important conclusions in differential and integral of fuzzy mapping [4, 5, 7, 11]. At same time, by the classical mathematical programming methods, the convexity of fuzzy mapping on convex sets and its application in fuzzy programming are discussed [1-3, 9], which greatly enriched the research contents of mathematical programming $[10,13,16]$.

Convex fuzzy planning is a kind of fuzzy programming problem, which objective function is convex fuzzy mapping (fuzzy convex function) and constraint set is convex set. In order to better use the analysis methods to discuss convex fuzzy programming problem, Wang and $\mathrm{Wu}$ [14] gave the concepts of the differential and gradient of fuzzy mapping in 2003. At the same time, the concept of sup-differential and sup-gradient of fuzzy mapping was first given, and the characterization theorem which the differentiability (sub-differentiability) of fuzzy mapping was gained; in addition, in the application of sub-differential in convex fuzzy planning, two important conclusions were obtained. But, the basic properties of the sub-differential of fuzzy mapping were not studied. So, in 2005, Zhang [16] gave some concepts of subgradient, sub-differential, differential of convex fuzzy mapping and so on, study the application of convex

\footnotetext{
*Corresponding author

Email address: byebed@163.com (Yu-E Bao)
} 
fuzzy planning, and obtained some corresponding important conclusions. Convex fuzzy mappings have a promising application prospect in fuzzy planning, but the difficulty of its application is that few fuzzy mappings have convexity. So the convex extension of normal fuzzy mapping is such a very meaningful work, which enables us to study of optimization problem on the fuzzy mapping by the principle of fuzzy convex analysis.

On convexification for fuzzy mapping, a method of changing normal fuzzy mapping into convex fuzzy mapping is given via the epigraph of fuzzy mapping [8, 17], and a characterization theorem of convex fuzzy mapping is obtained. The second purpose of this paper is to study convex extension problem of fuzzy mapping by means of the methods of convex fuzzy mapping. Firstly, in Section 3, we discuss some basic properties of differential and sub-differential (referred to the local sub-differential in this paper) of fuzzy mapping given by Wang and Wu [14]. Secondly, in the fourth section, the concept of sub-differentiability of fuzzy mapping is generalized, and its basic properties are discussed. At last, in Section 4, some convex continuation problems of fuzzy mapping are studied, a sufficient condition that the convex extension of fuzzy mapping exists is gained.

\section{Preliminaries}

First, we recall some definitions and results about fuzzy numbers (see [14, 15]).

Let $R$ be the real numbers field. A fuzzy set $u$ on $R$ is called a fuzzy number, if it has the following properties:

(1) $u$ is upper semi-continuous;

(2) $u$ is normal, i.e., there exists an $x_{0} \in R$ such that $u\left(x_{0}\right)=1$;

(3) $u$ is convex, i.e., $u(\lambda x+(1-\lambda) y) \leqslant \min ((u(x), u(y))$ whenever $x, y \in R$ and $\lambda \in[0,1]$;

(4) $[u]^{0}=\overline{\{x \mid u(x)>0\}}$ is a compact set.

Let $\mathcal{F}_{0}$ denote the family of all fuzzy numbers and $\mathcal{F}_{0}$ is called fuzzy number space.

For any $\alpha \in R$, define a fuzzy number $\widetilde{\alpha}$ by

$$
\tilde{\alpha}(t)= \begin{cases}1, & t=\alpha \\ 0, & t \neq \alpha,\end{cases}
$$

for any $t \in R$.

For $r \in[0,1]$, the $r$-level set of fuzzy number $u$ is the nonempty bounded closed interval $[u]^{r}=$ $\left[u_{*}(r), u^{*}(r)\right]$.

We call

$$
u=\left\{\left(u_{*}(r), u^{*}(r), r\right) \mid 0 \leqslant r \leqslant 1\right\}
$$

is the parametric expression of $u$.

For any $u, v \in \mathcal{F}_{0}$ and $\lambda \in R$, the addition and scalar multiplication on $\mathcal{F}_{0}$ can be represented as:

$$
\begin{aligned}
u+v & =\left\{\left(u_{*}(r)+v_{*}(r), u^{*}(r)+v^{*}(r), r\right) \mid 0 \leqslant r \leqslant 1\right\}, \\
\lambda u & = \begin{cases}\left\{\left(\lambda u_{*}(r), \lambda u^{*}(r), r\right) \mid o \leqslant r \leqslant 1\right\}, & \lambda \geqslant 0, \\
\left\{\left(\lambda u^{*}(r), \lambda u_{*}(r), r\right) \mid o \leqslant r \leqslant 1\right\}, & \lambda<0,\end{cases}
\end{aligned}
$$

for any $r \in[0,1]$.

$$
\begin{aligned}
(u+v)_{*}(r) & =u_{*}(r)+v_{*}(r),(u+v)^{*}(r)=u^{*}(r)+v^{*}(r), \\
(\lambda u)_{*}(r) & = \begin{cases}\lambda u_{*}(r), & \lambda \geqslant 0 \\
\lambda u^{*}(r), & \lambda>0\end{cases}
\end{aligned}
$$




$$
(\lambda u)^{*}(r)= \begin{cases}\lambda u^{*}(r), & \lambda \geqslant 0 \\ \lambda u_{*}(r), & \lambda>0\end{cases}
$$

For $u, v \in \mathcal{F}_{0}$, if

$$
u_{*}(r) \leqslant v_{*}(r) \text { and } u^{*}(r) \leqslant v^{*}(r) \text { for any } r \in[0,1] \text {, }
$$

then we call $u \leqslant v$.

Theorem 2.1 ([14]). Let $u, v, w \in \mathcal{F}_{0}$.

(1) $u \leqslant v \Longleftrightarrow u+w \leqslant v+w$;

(2) $u \leqslant v \Longrightarrow \lambda u \leqslant \lambda v, \lambda \geqslant 0 ; u \leqslant v \Longrightarrow \lambda u \leqslant \lambda u, \lambda<0$.

For $u, v \in \mathcal{F}_{0}$, define

$$
\mathrm{D}(\mathrm{u}, v)=\sup _{\mathrm{r} \in[0,1]} \max \left\{\left|\mathrm{u}_{*}(\mathrm{r})-v_{*}(\mathrm{r})\right|,\left|\mathrm{u}^{*}(\mathrm{r})-v^{*}(\mathrm{r})\right|\right\}
$$

then $\left(\mathcal{F}_{0}, \mathrm{D}\right)$ is a complete metric space, and satisfies

$$
\mathrm{D}(\mathrm{u}+w, v+w)=\mathrm{D}(\mathrm{u}, v), \mathrm{D}(\lambda \mathrm{u}, \lambda v)=|\lambda| \mathrm{D}(\mathrm{u}, v),
$$

for any $u, v, w \in \mathcal{F}_{0}, \lambda \in R$.

A subset $\mathrm{H}$ of $\mathcal{F}_{0}$ is said to be a lower bound if there exists a fuzzy number $v \in \mathcal{F}_{0}$, such that $u \geqslant v$ for any $u \in \mathrm{H}$. $v_{0} \in \mathcal{F}_{0}$ is called the infimum of $\mathrm{H}$, if $v_{0}$ is a lower bound of $\mathrm{H}$ and satisfies $v_{0} \geqslant v$ for any lower bound $v$ of $\mathrm{H}$, and we denote it as $v_{0}=\inf \{\mathrm{u} \mid \mathrm{u} \in \mathrm{H}\}$.

Theorem 2.2 ([15]). Let a subset $M$ of $\mathcal{F}_{0}$ is lower bounded, then its infimum must exist.

For $u_{i} \in \mathcal{F}_{0}, u_{i}=\left\{\left(u_{i *}(r), u_{i}^{*}(r), r\right) \mid 0 \leqslant r \leqslant 1\right\}(i=1,2, \cdots, n)$, we define

$$
u=\left(u_{1}, u_{2}, \cdots, u_{n}\right)
$$

is $n$-dimensional fuzzy vector in $\mathcal{F}_{0}$, the set of all $n$-dimensional fuzzy vectors is denoted by $\mathcal{F}_{0}^{n}$.

Let $\mathrm{R}^{\mathrm{n}}$ denotes $\mathrm{n}$-dimensional Euclidean space. For

$$
x=\left(x_{1}, x_{2}, \cdots, x_{n}\right), y=\left(y_{1}, y_{2}, \cdots, y_{n}\right) \in R^{n},
$$

we define $x \leqslant y$ if and only if $x_{i} \leqslant y_{i}(i=1,2, \cdots, n) . d(x, y)$ is the Euclidean distance between $x$ and $y$.

For $u=\left(u_{1}, u_{2}, \cdots, u_{n}\right), v=\left(v_{1}, v_{2}, \cdots, v_{n}\right) \in \mathcal{F}_{0}^{n}$ and $\lambda \in R$, the addition and scalar multiplication on $\mathcal{F}_{0}^{\mathrm{n}}$ are defined by

$$
u+v=\left(u_{1}+v_{1}, u_{2}+v_{2}, \cdots, u_{n}+v_{n}\right), \lambda u=\left(\lambda u_{1}, \lambda u_{2}, \cdots, \lambda u_{n}\right),
$$

and we define $u=v$ if and only if $u_{i}=v_{i}(i=1,2, \cdots, n)$.

Theorem 2.3. Let mapping $\mathrm{D}_{\mathrm{n}}: \mathcal{F}_{0}^{\mathrm{n}} \times \mathcal{F}_{0}^{\mathrm{n}} \longrightarrow[0,+\infty)$ be defined by

$$
D_{n}(u, v)=\left(\sum_{i=1}^{n}\left(D\left(u_{i}, v_{i}\right)\right)^{p}\right)^{\frac{1}{p}}(p \geqslant 1) .
$$

Then

(1) $\mathrm{D}_{\mathrm{n}}(\mathrm{u}, v)=\mathrm{D}_{\mathrm{n}}(v, \mathrm{u})$; 
(2) $D_{n}(u, v) \geqslant 0$;

(3) $\mathrm{D}_{\mathrm{n}}(\mathrm{u}, \mathrm{v})=0 \Longleftrightarrow \mathrm{u}=v$;

(4) $D_{n}(u, v) \leqslant D_{n}(u, w)+D_{n}(w, v)$ ，

where

$$
D\left(u_{i}, v_{i}\right)=\sup _{r \in[0,1]} \max \left\{\left|u_{i *}(r)-v_{i *}(r)\right|,\left|u_{i}^{*}(r)-v_{i}^{*}(r)\right|\right\} .
$$

Proof. (1) and (2) obviously are established.

(3) $\mathrm{D}_{\mathrm{n}}(\mathrm{u}, v)=0 \Longleftrightarrow \mathrm{D}\left(\mathrm{u}_{\mathrm{i}}, v_{\mathrm{i}}\right)=0(\mathrm{i}=1,2, \cdots, \mathrm{n}) \Longleftrightarrow \mathrm{u}_{\mathrm{i}}=v_{\mathrm{i}} \Longleftrightarrow \mathrm{u}=v$.

(4) By Minkowski inequality,

$$
\begin{aligned}
D_{n}(u, v) & =\left(\sum_{i=1}^{n}\left(D\left(u_{i}, v_{i}\right)\right)^{p}\right)^{\frac{1}{p}} \\
& \leqslant\left(\sum_{i=1}^{n}\left(D\left(u_{i}, w_{i}\right)+D\left(w_{i}, v_{i}\right)\right)^{p}\right)^{\frac{1}{p}} \\
& \leqslant\left(\sum_{i=1}^{n}\left(D\left(u_{i}, w_{i}\right)\right)^{p}\right)^{\frac{1}{p}}+\left(\sum_{i=1}^{n}\left(D\left(w_{i}, v_{i}\right)\right)^{p}\right)^{\frac{1}{p}} \leqslant D_{n}(u, w)+D_{n}(w, v),
\end{aligned}
$$

hence mapping $D_{n}$ is the distance on $\mathcal{F}_{0}^{n}$.

In this paper, the fuzzy mapping (fuzzy-valued function) is referred to the mapping projecting of a nonempty subset $M$ of $R^{n}$ to fuzzy number space $\mathcal{F}_{0}$, namely $F: M \rightarrow \mathcal{F}_{0}$. Form the parametric expression of fuzzy number, that fuzzy mapping can be expressed as:

$$
\mathrm{F}(\mathrm{x})=\left\{\left(\mathrm{F}(\mathrm{x})_{*}(\mathrm{r}), \mathrm{F}(\mathrm{x})^{*}(\mathrm{r}), \mathrm{r}\right) \mid 0 \leqslant \mathrm{r} \leqslant 1\right\},
$$

where for any $r \in[0,1], F(x)_{*}(r)$ and $F(x)^{*}(r)$ are defined as real-valued functions on $M$.

A fuzzy mapping $F: M \rightarrow \mathcal{F}_{0}$ is called to be lower bound if there exists a fuzzy number $v \in \mathcal{F}_{0}$, such that $F(u) \geqslant v$ for any $u \in M$.

Definition 2.4 ([17]). Let $F: M \rightarrow \mathcal{F}_{0}$ be a fuzzy mapping, $G=\operatorname{Co}($ epi( $F)$ ), then the fuzzy mapping defined on $\mathrm{CoM}$

$$
\mathrm{F}_{\mathrm{C}}(\mathrm{x})=\inf \left\{\mathrm{u} \mid(x, \mathrm{u}) \in \mathrm{G}, \mathrm{u} \in \mathcal{F}_{0}\right\}
$$

is called the convex hull of $F$, denoted by $F_{C}=\mathrm{CoF}$, where

$$
\operatorname{epi}(F)=\left\{(x, u) \mid x \in M, u \in \mathcal{F}_{0}, F(x) \leqslant u\right\}
$$

is the epigraph of $F, C o M$ and $\operatorname{Co}($ epi $(F))$ are the convex hulls corresponding to $M$ and epi(F), respectively.

We can easily prove that $F_{C}=C_{o} F$ is the convex fuzzy mapping on $C_{0} M$, and $F_{C}$ is called the convexification fuzzy mapping of $\mathrm{F}$.

Theorem 2.5 ([17]). Let $\mathrm{F}_{\mathrm{C}}$ be the convexification fuzzy mapping of fuzzy mapping $\mathrm{F}: \mathrm{M} \rightarrow \mathcal{F}_{0}$, then

$$
\begin{aligned}
F_{C}(x) & =\inf _{m \in N}\left\{\lambda_{1} F\left(x^{1}\right)+\lambda_{2} F\left(x^{2}\right)+\cdots+\lambda_{m} F\left(x^{m}\right) \mid \lambda_{j}>0,\right. \\
x^{j} & \left.\in M(j=1,2, \cdots, m), \sum_{j=1}^{m}=1, \sum_{j=1}^{m} \lambda_{j} x^{j}=x\right\} .
\end{aligned}
$$


Definition 2.6 ([14]). Let $F: M \rightarrow \mathcal{F}_{0}$ be a fuzzy mapping, $x^{0}=\left(x_{1}^{0}, x_{2}^{0}, \cdots, x_{n}^{0}\right) \in \operatorname{int}(M)$. If there exists an $\mathrm{u}=\left(\mathrm{u}_{1}, \mathrm{u}_{2}, \cdots, \mathrm{u}_{\mathrm{n}}\right) \in \mathcal{F}_{0}^{\mathfrak{n}}$, such that

$$
\lim _{x \rightarrow x^{0}} \frac{D\left(F(x)+\sum_{x_{i}<x_{i}^{0}}\left|x_{i}-x_{i}^{0}\right| u_{i}, F\left(x^{0}\right)+\sum_{x_{i} \geqslant x_{i}^{0}}\left|x_{i}-x_{i}^{0}\right| u_{i}\right)}{d\left(x, x^{0}\right)}=0,
$$

then we say $F$ to be differentiable at $x^{0}$, and call $\left(u_{1}, u_{2}, \cdots, u_{n}\right)\left(\right.$ denoted by $\left.\nabla F\left(x_{0}\right)=\left(u_{1}, u_{2}, \cdots, u_{n}\right)\right)$ the gradient of $F$ at $x^{0}$, where $x=\left(x_{1}, x_{2}, \cdots, x_{n}\right)$.

Definition 2.7 ([15]). Let $F: M \rightarrow \mathcal{F}_{0}$ be a fuzzy mapping. $F$ is lower semi-continuous at a point $x^{0}$ if for any $\varepsilon>0$, there exists $\delta>0$ such that

$$
F\left(x^{0}\right) \leqslant F(x)+\widetilde{\varepsilon}
$$

holds for all $x \in M$ and $\left\|x-x^{0}\right\|<\delta$. $F$ is called to be lower semi-continuous on $M$ if it is lower semi-continuous at every point of $M$.

Theorem 2.8. If $\mathrm{F}$ be the lower semi-continuous fuzzy mapping on $\mathrm{M}$, then $\mathrm{F}(\mathrm{x})_{*}(\mathrm{r})$ and $\mathrm{F}(\mathrm{x})^{*}(\mathrm{r})$ are lower semi-continuous real-valued functions on $M$ for any $r \in[0,1]$.

Proof. Let $\mathrm{F}$ be the lower semi-continuous fuzzy mapping on $M, x^{0} \in M$, then for any $\varepsilon>0$, there exists $\delta>0$ such that

$$
F\left(x^{0}\right) \leqslant F(x)+\widetilde{\varepsilon}
$$

holds for all $x \in M$ and $\left\|x-x^{0}\right\|<\delta$. Therefore, for any $r \in[0,1]$, we have

$$
\begin{aligned}
& \mathrm{F}\left(\mathrm{x}^{0}\right)_{*}(\mathrm{r}) \leqslant \mathrm{F}(\mathrm{x})_{*}(\mathrm{r})+\varepsilon, \\
& \mathrm{F}\left(\mathrm{x}^{0}\right)^{*}(\mathrm{r}) \leqslant \mathrm{F}(\mathrm{x})^{*}(\mathrm{r})+\varepsilon .
\end{aligned}
$$

It follows that $F(x)_{*}(r)$ and $F(x)^{*}(r)$ are lower semi-continuous fuzzy-valued functions on $M$.

Remark 2.9. Let $f: M \rightarrow(-\infty,+\infty)$ be a real-valued function, $x^{0} \in M$, then the following conditions are equivalent:

(1) $f$ is lower semi-continuous at point $x^{0}$;

(2) for any $\varepsilon>0$, there exists $\delta>0$ such that $f\left(x^{0}\right) \leqslant f(x)+\varepsilon$, holds for all $x \in M$ and $\left\|x-x^{0}\right\|<\delta$.

(3) for any $x_{n} \in M(n=1,2, \cdots)$, if $\left\|x-x^{0}\right\| \rightarrow 0(n \rightarrow \infty)$, then

$$
f\left(x^{0}\right) \leqslant \underline{\lim }_{n \rightarrow \infty} f\left(x_{n}\right) .
$$

Related concepts of real function can be found in [12].

\section{The differentiability and local sub-differentiability of the fuzzy mapping}

In this section, we discuss some basic properties of the differential and local sub-differential of the fuzzy mapping.

Theorem 3.1. Let $\mathrm{F}, \mathrm{G}$ be fuzzy mappings $\left(\mathrm{M} \rightarrow \mathcal{F}_{0}\right)$, and be differentiable at point $x^{0} \in \operatorname{int}(\mathrm{M})$, then $\lambda \mathrm{F}(\lambda>0)$ and $\mathrm{F}+\mathrm{G}$ are differentiable at point $\chi^{0}$, and

$$
\nabla(\lambda F)\left(x^{0}\right)=\lambda \nabla F\left(x^{0}\right), \nabla(F+G)\left(x^{0}\right)=\nabla F\left(x^{0}\right)+\nabla G\left(x^{0}\right) .
$$


Proof. (1) Let $\nabla \mathrm{F}\left(\mathrm{x}_{0}\right)=\left(\mathrm{u}_{1}, \mathrm{u}_{2}, \cdots, \mathrm{u}_{\mathrm{n}}\right)$, then

$$
\begin{aligned}
\lim _{x \rightarrow x_{0}} & \frac{D\left(\lambda F(x)+\sum_{x_{i}<x_{i}^{0}}\left|x_{i}-x_{i}^{0}\right|\left(\lambda u_{i}\right), \lambda F\left(x^{0}\right)+\sum_{x_{i} \geqslant x_{i}^{0}}\left|x_{i}-x_{i}^{0}\right|\left(\lambda u_{i}\right)\right)}{d\left(x, x^{0}\right)} \\
& =\lambda \lim _{x \rightarrow x_{0}} \frac{D\left(F(x)+\sum_{x_{i}<x_{i}^{0}}\left|x_{i}-x_{i}^{0}\right|\left(u_{i}\right), F\left(x_{0}\right)+\sum_{x_{i} \geqslant x_{i}^{0}}\left|x_{i}-x_{i}^{0}\right|\left(u_{i}\right)\right)}{d\left(x, x^{0}\right)}=0 .
\end{aligned}
$$

Therefore, we have

$$
\nabla(\lambda F)\left(x^{0}\right)=\left(\lambda u_{1}, \lambda u_{2}, \cdots, \lambda u_{n}\right) .
$$

So

$$
\nabla(\lambda F)\left(x^{0}\right)=\lambda \nabla F\left(x^{0}\right) .
$$

(2) Let $\nabla F\left(x^{0}\right)=\left(u_{1}, u_{2}, \cdots, u_{n}\right)$, then

$$
\begin{aligned}
\lim _{x \rightarrow x_{0}} & \frac{D\left(F(x)+\sum_{x_{i}<x_{i}^{0}}\left|x_{i}-x_{i}^{0}\right|\left(u_{i}\right), F\left(x_{0}\right)+\sum_{x_{i} \geqslant x_{i}^{0}}\left|x_{i}-x_{i}^{0}\right|\left(u_{i}\right)\right)}{d\left(x, x^{0}\right)} \\
& =\lim _{x \rightarrow x^{0}} \sup _{r \in[0,1]} \max \left\{\frac{\left|\left(F(x)+\sum_{x_{i}<x_{i}^{0}}\left(x_{i}^{0}-x_{i}\right) u_{i}\right)_{*}(r)-\left(F\left(x^{0}\right)+\sum_{x_{i} \geqslant x_{i}^{0}}\left(x_{i}-x_{i}^{0}\right) u_{i}\right)_{*}(r)\right|}{d\left(x, x^{0}\right)},\right. \\
& \left.\frac{\left|\left(F(x)+\sum_{x_{i}<x_{i}^{0}}\left(x_{i}^{0}-x_{i}\right) u_{i}\right)^{*}(r)-\left(F\left(x^{0}\right)+\sum_{x_{i} \geqslant x_{i}^{0}}\left(x_{i}-x_{i}^{0}\right) u_{i}\right)^{*}(r)\right|}{d\left(x, x^{0}\right)}\right\}=0 .
\end{aligned}
$$

Therefore, we have

$$
\begin{aligned}
& \lim _{x \rightarrow x^{0}} \sup _{r \in[0,1]} \frac{\left|F(x)_{*}(r)-F\left(x^{0}\right)_{*}(r)-\sum_{i=1}^{n}\left(x_{i}-x_{i}^{0}\right) u_{i *}(r)\right|}{d\left(x, x^{0}\right)}=0, \\
& \lim _{x \rightarrow x^{0}} \sup _{r \in[0,1]} \frac{\left|F(x)^{*}(r)-F\left(x^{0}\right)^{*}(r)-\sum_{i=1}^{n}\left(x_{i}-x_{i}^{0}\right) u_{i}^{*}(r)\right|}{d\left(x, x^{0}\right)}=0 .
\end{aligned}
$$

Let $\nabla \mathrm{G}\left(\mathrm{x}^{0}\right)=\left(v_{1}, v_{2}, \cdots, v_{\mathrm{n}}\right)$, then can similarly obtain

$$
\begin{aligned}
& \lim _{x \rightarrow x^{0}} \sup _{r \in[0,1]} \frac{\left|G(x)_{*}(r)-G\left(x^{0}\right)_{*}(r)-\sum_{i=1}^{n}\left(x_{i}-x_{i}^{0}\right) v_{i *}(r)\right|}{d\left(x, x^{0}\right)}=0, \\
& \lim _{x \rightarrow x^{0}} \sup _{r \in[0,1]} \frac{\left|G(x)^{*}(r)-G\left(x^{0}\right)^{*}(r)-\sum_{i=1}^{n}\left(x_{i}-x_{i}^{0}\right) v_{i}^{*}(r)\right|}{d\left(x, x^{0}\right)}=0 .
\end{aligned}
$$

So

$$
\begin{aligned}
& \sup _{r \in[0,1]} \frac{\left|\left(F(x)_{*}(r)+G(x)_{*}(r)\right)-\left(F\left(x^{0}\right)_{*}(r)+G\left(x^{0}\right)_{*}(r)\right)-\sum_{i=1}^{n}\left(x_{i}-x_{i}^{0}\right)\left(u_{i *}(r)+v_{i *}(r)\right)\right|}{d\left(x, x^{0}\right)} \\
& \leqslant \sup _{r \in[0,1]} \frac{\left|F(x)_{*}(r)-F\left(x^{0}\right)_{*}(r)-\sum_{i=1}^{n}\left(x_{i}-x_{i}^{0}\right) u_{i *}(r)\right|}{d\left(x, x^{0}\right)} \\
& \quad+\sup _{r \in[0,1]} \frac{\left|G(x)_{*}(r)-G\left(x^{0}\right)_{*}(r)-\sum_{i=1}^{n}\left(x_{i}-x_{i}^{0}\right) v_{i *}(r)\right|}{d\left(x, x^{0}\right)}, \\
& \sup _{r \in[0,1]} \frac{\left|\left(F(x)^{*}(r)+G(x)^{*}(r)\right)-\left(F\left(x^{0}\right)^{*}(r)+G\left(x^{0}\right)^{*}(r)\right)-\sum_{i=1}^{n}\left(x_{i}-x_{i}^{0}\right)\left(u_{i}^{*}(r)+v_{i}^{*}(r)\right)\right|}{d\left(x, x^{0}\right)}
\end{aligned}
$$




$$
\begin{aligned}
& \leqslant \sup _{r \in[0,1]} \frac{\left|F(x)^{*}(r)-F\left(x^{0}\right)^{*}(r)-\sum_{i=1}^{n}\left(x_{i}-x_{i}^{0}\right) u_{i}^{*}(r)\right|}{d\left(x, x^{0}\right)} \\
& \quad+\sup _{r \in[0,1]} \frac{\left|G(x)^{*}(r)-G\left(x^{0}\right)^{*}(r)-\sum_{i=1}^{n}\left(x_{i}-x_{i}^{0}\right) v_{i}^{*}(r)\right|}{d\left(x, x^{0}\right)} .
\end{aligned}
$$

Thus we can obtain

$$
\begin{aligned}
0 \leqslant & \lim _{x \rightarrow x^{0}} D\left((F+G)(x)+\sum_{x_{i}<x_{i}^{0}}\left|x_{i}-x_{i}^{0}\right|\left(u_{i}+v_{i}\right),(F+G)\left(x^{0}\right)+\sum_{x_{i} \geqslant x_{i}^{0}}\left(x_{i}-x_{i}^{0}\right)\left(u_{i}+v_{i}\right)\right) \\
\leqslant & \lim _{x \rightarrow x^{0}} D\left(F(x)+\sum_{x_{i}<x_{i}^{0}}\left|x_{i}-x_{i}^{0}\right| u_{i}, F\left(x^{0}\right)+\sum_{x_{i} \geqslant x_{i}^{0}}\left(x_{i}-x_{i}^{0}\right) u_{i}\right) \\
& +\lim _{x \rightarrow x^{0}} D\left(G(x)+\sum_{x_{i}<x_{i}^{0}}\left|x_{i}-x_{i}^{0}\right| u_{i}, G\left(x^{0}\right)+\sum_{x_{i} \geqslant x_{i}^{0}}\left(x_{i}-x_{i}^{0}\right) v_{i}\right)=0 .
\end{aligned}
$$

Therefore,

$$
\lim _{x \rightarrow x^{0}} D\left((F+G)(x)+\sum_{x_{i}<x_{i}^{0}}\left|x_{i}-x_{i}^{0}\right|\left(u_{i}+v_{i}\right),(F+G)\left(x^{0}\right)+\sum_{x_{i} \geqslant x_{i}^{0}}\left(x_{i}-x_{i}^{0}\right)\left(u_{i}+v_{i}\right)\right)=0 .
$$

So

$$
\nabla(\mathrm{F}+\mathrm{G})\left(\mathrm{x}^{0}\right)=\left(\mathrm{u}_{1}+v_{1}, \mathrm{u}_{2}+v_{2}, \cdots, \mathrm{u}_{\mathfrak{n}}+v_{\mathrm{n}}\right)=\nabla \mathrm{F}\left(\mathrm{x}^{0}\right)+\nabla \mathrm{G}\left(\mathrm{x}^{0}\right)
$$

Definition 3.2 ([10]). Let $F: M \rightarrow \mathcal{F}_{0}$ be a fuzzy mapping, $x^{0}=\left(x_{1}^{0}, x_{2}^{0}, \cdots, x_{n}^{0}\right) \in M$. If there exist $\mathrm{u}=\left(\mathrm{u}_{1}, \mathrm{u}_{2}, \cdots, \mathrm{u}_{\mathrm{n}}\right) \in \mathcal{F}_{0}^{\mathrm{n}}$ and $\delta>0$, such that

$$
F(x)+\sum_{x_{i}<x_{i}^{0}}\left|x_{i}-x_{i}^{0}\right| u_{i} \geqslant F\left(x^{0}\right)+\sum_{x_{i} \leqslant x_{i}^{0}}\left(x_{i}-x_{i}^{0}\right) u_{i}
$$

holds for all $x \in U\left(x^{0}, \delta\right) \cap M$ (where $\left.U\left(x^{0}, \delta\right)=\left\{x \in R^{n} \mid d\left(x, x^{0}\right)<\delta\right\}\right)$, then we call $u=\left(u_{1}, u_{2}, \cdots, u_{n}\right) a$ sub-gradient at $x^{0}$ of $F$, and say the set of all sub-gradients of to be sub-differential of $F$ at $x^{0}$ of $F$ (denoted by $\left.\partial F\left(x^{0}\right)\right)$, i.e.,

$$
\partial F\left(x^{0}\right)=\left\{\xi \mid u=\left(u_{1}, u_{2}, \cdots, u_{n}\right) \in \mathcal{F}_{0}^{\mathfrak{n}}\right.
$$

and there exists $\delta>0$, such that

$$
\left.F(x)+\sum_{x_{i}<x_{i}^{0}}\left|x_{i}-x_{i}^{0}\right| u_{i} \geqslant F\left(x^{0}\right)+\sum_{x_{i} \geqslant x_{i}^{0}}\left(x_{i}-x_{i}^{0}\right) u_{i} \text { for any } x \in U\left(x^{0}, \delta\right) \cap M\right\} .
$$

Remark 3.3. In this paper, we define the sub-differential of Definition 3.2 that is local sub-differential at $x^{0}$, denoted as $\hat{\partial} F\left(x^{0}\right)$.

Theorem 3.4. Let $\mathrm{F}_{i}: M \rightarrow \mathcal{F}_{0}(i=1,2)$ be fuzzy mappings, $x^{0} \in M$, then

(1) $\hat{\partial}(\lambda F)\left(\chi^{0}\right)=\lambda \hat{\partial} F\left(x^{0}\right)$ for any $\lambda>0$;

(2) $\hat{\partial} F_{1}\left(x^{0}\right)+\hat{\partial} F_{2}\left(x^{0}\right) \subset \hat{\partial}\left(F_{1}+F_{2}\right)\left(x^{0}\right)$. 
Proof. (1) For $u=\left(u_{1}, u_{2}, \cdots, u_{n}\right) \in \hat{\partial}(\lambda F)\left(x^{0}\right)$, by Definition 3.2, there exists $\delta>0$, such that

$$
\lambda F(x)+\sum_{x_{i}<x_{i}^{0}}\left|x_{i}-x_{i}^{0}\right| u_{i} \geqslant \lambda F\left(x^{0}\right)+\sum_{x_{i} \geqslant x_{i}^{0}}\left(x_{i}-x_{i}^{0}\right) u_{i},
$$

for any $x=\left(x_{1}, x_{2}, \cdots, x_{n}\right) \in \mathrm{U}\left(x^{0}, \delta\right) \cap M$.

By $\lambda>0$, we have

$$
F(x)+\sum_{x_{i}<x_{i}^{0}}\left|x_{i}-x_{i}^{0}\right|\left(\frac{u_{i}}{\lambda}\right) \geqslant F\left(x^{0}\right)+\sum_{x_{i} \geqslant x_{i}^{0}}\left(x_{i}-x_{i}^{0}\right)\left(\frac{u_{i}}{\lambda}\right) .
$$

Therefore,

$$
\left(\frac{u_{1}}{\lambda}, \frac{u_{2}}{\lambda}, \cdots, \frac{u_{n}}{\lambda}\right)=\frac{1}{\lambda}\left(u_{1}, u_{2}, \cdots, u_{n}\right) \in \hat{\partial} F\left(x^{0}\right) .
$$

So $u=\left(u_{1}, u_{2}, \cdots, u_{n}\right) \in \lambda \hat{\partial} F\left(x^{0}\right)$, i.e., $\hat{\partial}(\lambda F)\left(x^{0}\right) \in \lambda \hat{\partial} F\left(x^{0}\right)$.

We can similarly obtain $\lambda \hat{\partial} F\left(x^{0}\right) \subset \hat{\partial}(\lambda F)\left(x^{0}\right)$. So $\lambda \hat{\partial} F\left(x^{0}\right)=\hat{\partial}(\lambda F)\left(x^{0}\right)$.

(2) Let $u \in \hat{\partial} F_{1}\left(x^{0}\right)+\hat{\partial} F_{2}\left(x^{0}\right)$, then there exist $u^{1} \in \hat{\partial} F_{1}\left(x^{0}\right), u^{2} \in \hat{\partial} F_{2}\left(x^{0}\right)$, such that $u=u^{1}+u^{2}$. By Definition 3.2, there exist $\delta_{1}, \delta_{2}>0$, such that

$$
F_{1}(x)+\sum_{x_{i}<x_{i}^{0}}\left|x_{i}-x_{i}^{0}\right| u_{i}^{1} \geqslant F_{1}\left(x^{0}\right)+\sum_{x_{i} \geqslant x_{i}^{0}}\left|x_{i}-x_{i}^{0}\right| u_{i}^{1}
$$

for any $x \in \mathrm{U}\left(\mathrm{x}^{0}, \delta_{1}\right) \cap \mathrm{M}$,

$$
F_{2}(x)+\sum_{x_{i}<x_{i}^{0}}\left|x_{i}-x_{i}^{0}\right| u_{i}^{2} \geqslant F_{2}\left(x^{0}\right)+\sum_{x_{i} \geqslant x_{i}^{0}}\left|x_{i}-x_{i}^{0}\right| u_{i}^{2},
$$

for any $x \in \mathrm{U}\left(\mathrm{x}^{0}, \delta_{2}\right) \cap \mathrm{M}$.

Take $\delta=\min \left\{\delta_{1}, \delta_{2}\right\}$, then by (3.1) and (3.2) we have

$$
\left(F_{1}+F_{2}\right)(x)+\sum_{x_{i}<x_{i}^{0}}\left|x_{i}-x_{i}^{0}\right|\left(u_{i}^{1}+u_{i}^{2}\right) \geqslant\left(F_{1}+F_{2}\right)\left(x^{0}\right)+\sum_{x_{i} \geqslant x_{i}^{0}}\left(x_{i}-x_{i}^{0}\right)\left(u_{i}^{1}+u_{i}^{2}\right),
$$

for any $x \in \mathrm{U}\left(x^{0}, \delta\right) \cap M$.

Therefore, we have

$$
u=u^{1}+u^{2} \in \hat{\partial}\left(F_{1}+F_{2}\right)\left(x^{0}\right) .
$$

So

$$
\hat{\partial} F_{1}\left(x^{0}\right)+\hat{\partial} F_{2}\left(x^{0}\right) \subset \hat{\partial}\left(F_{1}+F_{2}\right)\left(x^{0}\right)
$$

Corollary 3.5. If there exists $\alpha>0$, such that $\mathrm{F}_{1}(\mathrm{x})=\alpha \mathrm{F}_{2}(\mathrm{x})$ for any $\mathrm{x} \in \mathrm{M}$, then

$$
\hat{\partial}\left(F_{1}+F_{2}\right)\left(x^{0}\right)=\hat{\partial} F_{1}\left(x^{0}\right)+\hat{\partial} F_{2}\left(x^{0}\right) .
$$

Proof. By Theorem 3.4 we only need to prove

$$
\hat{\partial}\left(F_{1}+F_{2}\right)\left(x^{0}\right) \subset \hat{\partial} F_{1}\left(x^{0}\right)+\hat{\partial} F_{2}\left(x^{0}\right) .
$$

Let $u=\left(u_{1}, u_{2}, \cdots, u_{n}\right) \in \hat{\partial}\left(F_{1}+F_{2}\right)\left(x^{0}\right)$, then there exists $\delta>0$, such that

$$
\left(F_{1}+F_{2}\right)(x)+\sum_{x_{i}<x_{i}^{0}}\left|x_{i}-x_{i}^{0}\right| u_{i} \geqslant\left(F_{1}+F_{2}\right)\left(x^{0}\right)+\sum_{x_{i} \geqslant x_{i}^{0}}\left(x_{i}-x_{i}^{0}\right) u_{i},
$$


for any $x \in \mathrm{U}\left(\mathrm{x}^{0}, \delta\right) \cap M$.

By $F_{1}(x)=\alpha F_{2}(x)$, for any $x \in M$ we have

$$
\begin{aligned}
& (1+\alpha) F_{2}(x)+\sum_{x_{i}<x_{i}^{0}}\left|x_{i}-x_{i}^{0}\right| u_{i} \geqslant(1+\alpha) F_{2}\left(x^{0}\right)+\sum_{x_{i} \geqslant x_{i}^{0}}\left|x_{i}-x_{i}^{0}\right| u_{i}, \\
& \left(1+\frac{1}{\alpha}\right) F_{1}(x)+\sum_{x_{i}<x_{i}^{0}}\left|x_{i}-x_{i}^{0}\right| u_{i} \geqslant\left(1+\frac{1}{\alpha}\right) F_{1}\left(x^{0}\right)+\sum_{x_{i} \geqslant x_{i}^{0}}\left|x_{i}-x_{i}^{0}\right| u_{i},
\end{aligned}
$$

for any $x \in \mathrm{U}\left(\mathrm{x}^{0}, \delta\right) \cap \mathrm{M}$.

Therefore,

$$
\begin{aligned}
& u^{1}=\left(\frac{1}{1+\alpha} u_{1}, \frac{1}{1+\alpha} u_{2}, \cdots, \frac{1}{1+\alpha} u_{n}\right) \in \hat{\partial} F_{2}\left(x^{0}\right), \\
& u^{2}=\left(\frac{\alpha}{1+\alpha} u_{1}, \frac{\alpha}{1+\alpha} u_{2}, \cdots, \frac{\alpha}{1+\alpha} u_{n}\right) \in \hat{\partial} F_{1}\left(x^{0}\right) .
\end{aligned}
$$

Thus, we obtain

$$
u=u^{1}+u^{2} \in \hat{\partial} F_{1}\left(x^{0}\right)+\hat{\partial} F_{2}\left(x^{0}\right) \text {, i.e., } \hat{\partial}\left(F_{1}+F_{2}\right)\left(x^{0}\right) \subset \hat{\partial} F_{1}\left(x^{0}\right)+\hat{\partial} F_{2}\left(x^{0}\right) .
$$

Theorem 3.6. Let $\mathrm{F}: M \rightarrow \mathcal{F}_{0}$ be a fuzzy mapping, $\mathrm{u}=\left(\mathrm{u}_{1}, \mathrm{u}_{2}, \cdots, \mathrm{u}_{\mathrm{n}}\right) \in \mathcal{F}^{\mathrm{n}}$, then $\mathrm{u} \in \hat{\partial} \mathrm{F}\left(\mathrm{x}^{0}\right)$ if and only if $\mathrm{u}_{*}(\mathrm{r}) \in \partial \hat{\mathrm{F}}\left(\mathrm{x}^{0}\right)_{*}(\mathrm{r})$ and $\mathrm{u}^{*}(\mathrm{r}) \in \partial \hat{\mathrm{F}}\left(\mathrm{x}^{0}\right)^{*}(\mathrm{r})$ for any $\in \mathrm{r}[0,1]$, where

$$
u_{*}(r)=\left(u_{1 *}(r), u_{2 *}(r), \cdots, u_{n *}(r)\right), u^{*}(r)=\left(u_{1}^{*}(r), u_{2}^{*}(r), \cdots, u_{n}^{*}(r)\right)
$$

for any $r \in[0,1]$.

Proof. Let $u \in \hat{\partial} F\left(x^{0}\right)$, then there exists $\delta>0$, such that

$$
\begin{aligned}
& F(x)+\sum_{x_{i}<x_{i}^{0}}\left|x_{i}-x_{i}^{0}\right| u_{i} \geqslant F\left(x^{0}\right)+\sum_{x_{i} \geqslant x_{i}^{0}}\left(x_{i}-x_{i}^{0}\right) u_{i} \text { for any } x \in U\left(x^{0}, \delta\right) \cap M \\
& \Longleftrightarrow \mathrm{F}(\mathrm{x})_{*}(\mathrm{r})+\sum_{x_{i}<x_{i}^{0}}\left|x_{i}-x_{i}^{0}\right| u_{i *}(r) \geqslant F\left(x^{0}\right)_{*}(r)+\sum_{x_{i} \geqslant x_{i}^{0}}\left(x_{i}-x_{i}^{0}\right) u_{i *}(r) \\
& F(x)^{*}(r)+\sum_{x_{i}<x_{i}^{0}}\left|x_{i}-x_{i}^{0}\right| u_{i}^{*}(r) \geqslant F\left(x^{0}\right)^{*}(r)+\sum_{x_{i} \geqslant x_{i}^{0}}\left(x_{i}-x_{i}^{0}\right) u_{i}^{*}(r) \\
& \Longleftrightarrow F(x)_{*}(r) \geqslant F\left(x^{0}\right)_{*}(r)+\sum_{i=1}^{n}\left(x_{i}-x_{i}^{0}\right) u_{i *}(r) \\
& F(x)^{*}(r) \geqslant F\left(x^{0}\right)^{*}(r)+\sum_{i=1}^{n}\left(x_{i}-x_{i}^{0}\right) u_{i}^{*}(r) \\
& \Longleftrightarrow \mathrm{F}(x)_{*}(r) \geqslant F\left(x^{0}\right)_{*}(r)+\left\langle x-x^{0}, u_{*}(r)\right\rangle \\
& \mathrm{F}(x)^{*}(r) \geqslant F\left(x^{0}\right)^{*}(r)+\left\langle x-x^{0}, u^{*}(r)\right\rangle \\
& \Longleftrightarrow u_{*}(r) \in \partial \hat{\mathrm{F}}\left(x^{0}\right)_{*}(r) \text { and } u^{*}(r) \in \partial \hat{\mathrm{F}}\left(x^{0}\right)^{*}(r) \text {, }
\end{aligned}
$$

for any $r \in[0,1]$.

Theorem 3.7. Let a fuzzy mapping $\mathrm{F}: \mathrm{M} \rightarrow \mathcal{F}_{0}$ be differentiable at $x^{0} \in \operatorname{int}(\mathrm{M})$, then $\mathrm{F}$ is local sub-differential at $x^{0}$, and $\nabla \mathrm{F}\left(x^{0}\right) \in \hat{\partial} \mathrm{F}\left(x^{0}\right)$. 
Proof. Let $F$ be differentiable at $x^{0}=\left(x_{1}^{0}, x_{2}^{0}, \cdots, x_{n}^{0}\right)$, then there exists $u=\left(u_{1}, u_{2}, \cdots, u_{n}\right) \in \mathcal{F}^{n}$, such that

$$
\lim _{x \rightarrow x^{0}} \frac{D\left(F(x)+\sum_{x_{i}<x_{i}^{0}}\left|x_{i}-x_{i}^{0}\right| u_{i}, F\left(x^{0}\right)+\sum_{x_{i} \geqslant x_{i}^{0}}\left(x_{i}-x_{i}^{0}\right) u_{i}\right)}{d\left(x, x^{0}\right)}=0 .
$$

So

$$
\begin{aligned}
& \lim _{x \rightarrow x^{0}} \sup _{r \in[0,1]} \max _{\{}\left\{\frac{\left|\left(F(x)+\sum_{x_{i}<x_{i}^{0}}\left|x_{i}-x_{i}^{0}\right| u_{i}\right)_{*}(r),\left(F\left(x^{0}\right)+\sum_{x_{i} \geqslant x_{i}^{0}}\left(x_{i}-x_{i}^{0}\right) u_{i}\right)_{*}(r)\right|}{d\left(x, x^{0}\right)},\right. \\
& \left.\quad \frac{\left|\left(F(x)+\sum_{x_{i}<x_{i}^{0}}\left|x_{i}-x_{i}^{0}\right| u_{i}\right)^{*}(r),\left(F\left(x^{0}\right)+\sum_{x_{i} \geqslant x_{i}^{0}}\left(x_{i}-x_{i}^{0}\right) u_{i}\right)^{*}(r)\right|}{d\left(x, x^{0}\right)}\right\}=0 \\
& \Longleftrightarrow \lim _{x \rightarrow x^{0}} \sup _{r \in[0,1]} \frac{\left|F(x)_{*}(r)-F\left(x^{0}\right)_{*}(r)-\sum_{i=1}^{n}\left(x_{i}-x_{i}^{0}\right) u_{i *}(r)\right|}{d\left(x, x^{0}\right)}=0, \\
& \quad \lim _{x \rightarrow x^{0}} \sup _{r \in[0,1]} \frac{\left|F(x)^{*}(r)-F\left(x^{0}\right)^{*}(r)-\sum_{i=1}^{n}\left(x_{i}-x_{i}^{0}\right) u_{i}^{*}(r)\right|}{d\left(x, x^{0}\right)}=0 \\
& \Longleftrightarrow \quad F(x)_{*}(r)=F\left(x^{0}\right)_{*}(r)+\sum_{i=1}^{n}\left(x_{i}-x_{i}^{0}\right) u_{i *}(r)+o\left(d\left(x, x^{0}\right)\right), \\
& F(x)^{*}(r)=F\left(x^{0}\right)^{*}(r)+\sum_{i=1}^{n}\left(x_{i}-x_{i}^{0}\right) u_{i}^{*}(r)+o\left(d\left(x, x^{0}\right)\right),
\end{aligned}
$$

for any $r \in[0,1]$.

Therefore, for any $r \in[0,1], F(x)_{*}(r)$ and $F(x)^{*}(r)$ all are $n$-element differentiable real-valued functions on $M$, and

$$
\nabla F\left(x^{0}\right)_{*}(r)=\left(u_{1 *}(r), u_{2 *}(r), \cdots, u_{n *}(r)\right), \nabla F\left(x^{0}\right)^{*}(r)=\left(u_{1}^{*}(r), u_{2}^{*}(r), \cdots, u_{n}^{*}(r)\right)
$$

So

$$
\begin{aligned}
& \hat{\partial} \mathrm{F}\left(x^{0}\right)_{*}(\mathrm{r})=\left\{\nabla \mathrm{F}\left(x^{0}\right)_{*}(\mathrm{r})\right\}, \\
& \hat{\partial} \mathrm{F}\left(x^{0}\right)^{*}(\mathrm{r})=\left\{\nabla \mathrm{F}\left(\mathrm{x}^{0}\right)^{*}(\mathrm{r})\right\},
\end{aligned}
$$

for any $r \in[0,1]$.

Therefore by Theorem 3.6, we have

$$
\nabla \mathrm{F}\left(x^{0}\right)=\left(u_{1}, u_{2}, \cdots, u_{\mathfrak{n}}\right) \in \hat{\partial} F\left(x^{0}\right) .
$$

Theorem 3.8. Let $\mathrm{F}: M \rightarrow \mathcal{F}_{0}$ be a fuzzy mapping, then $\hat{\mathrm{F}}(\mathrm{x})$ is convex set or empty set.

Proof. Let $\hat{\partial} F(x) \neq \varnothing$, then for any $u^{1}, u^{2} \in \hat{\partial} F(x)$ and $\lambda \in(0,1)$, by Theorem 3.4, we have

$$
(1-\lambda) u^{1} \in \hat{\partial}\left((1-\lambda) F_{1}\right)(x), \lambda u^{2} \in \hat{\partial}\left(\lambda F_{2}\right)(x) .
$$

Therefore, there exist $\delta_{1}>0, \delta_{2}>0$, such that

$$
(1-\lambda) F(y)+\sum_{y_{i}<x_{i}}\left|y_{i}-x_{i}\right|(1-\lambda) u_{i}^{1} \geqslant(1-\lambda) F(x)+\sum_{y_{i} \geqslant x_{i}}\left(y_{i}-x_{i}\right)(1-\lambda) u_{i}^{1}
$$

for any $y \in \mathrm{U}\left(\mathrm{x}_{0}, \delta_{1}\right) \cap \mathrm{M}$,

$$
\lambda F(y)+\sum_{y_{i}<x_{i}}\left|y_{i}-x_{i}\right| \lambda u_{i}^{2} \geqslant \lambda F(x)+\sum_{y_{i} \geqslant x_{i}}\left(y_{i}-x_{i}\right) \lambda u_{i}^{2}
$$


for any $\mathrm{y} \in \mathrm{U}\left(\mathrm{x}_{0}, \delta_{2}\right) \cap \mathrm{M}$.

Take $\delta=\min \left\{\delta_{1}, \delta_{2}\right\}$, then by (3.3) and (3.4) we have

$$
F(y)+\sum_{y_{i}<x_{i}}\left|y_{i}-x_{i}\right|\left[(1-\lambda) u_{i}^{1}+\lambda u_{i}^{2}\right] \geqslant F(x)+\sum_{y_{i} \geqslant x_{i}}\left(y_{i}-x_{i}\right)\left[(1-\lambda) u_{i}^{1}+\lambda u_{i}^{2}\right],
$$

for any $y \in U\left(x_{0}, \delta\right) \cap M$.

Therefore, we have $(1-\lambda) u^{1}+\lambda u^{2} \in \hat{\partial} F(x)$, i.e., $\hat{\partial} F(x)$ is convex set.

\section{The sub-differentiability of the fuzzy mapping}

In this section, in order to discuss the problem of convex extension of fuzzy mapping, we will do some little change on the previous concept of local sub-differential of fuzzy mapping, and give a new concept of the sub-differentiability of the fuzzy mapping.

Definition 4.1. Let $F: M \rightarrow \mathcal{F}_{0}$ be a fuzzy mapping, $x^{0}=\left(x_{1}^{0}, x_{2}^{0}, \cdots, x_{n}^{0}\right) \in M$. If there exists $u=$ $\left(u_{1}, u_{2}, \cdots, u_{n}\right) \in \mathcal{F}_{0}^{n}$, such that

$$
F(x)+\sum_{x_{i}<x_{i}^{0}}\left|x_{i}-x_{i}^{0}\right| u_{i} \geqslant F\left(x^{0}\right)+\sum_{x_{i} \geqslant x_{i}^{0}}\left|x_{i}-x_{i}^{0}\right| u_{i}
$$

holds for all $x=\left(x_{1}, x_{2}, \cdots, x_{n}\right) \in M$, then we call $u=\left(u_{1}, u_{2}, \cdots, u_{n}\right)$ a sub-gradient at $x^{0}$ of $F$, and say the set of all sub-gradients of $F$ at $x^{0}$ to be sub-differential of $x^{0}$ of $F$, i.e.,

$$
\partial F\left(x^{0}\right)=\left\{u\left|u \in \mathcal{F}_{0}^{n}, F(x)+\sum_{x_{i}<x_{i}^{0}}\right| x_{i}-x_{i}^{0} \mid u_{i} \geqslant F\left(x^{0}\right)+\sum_{x_{i} \geqslant x_{i}^{0}}\left(x_{i}-x_{i}^{0}\right) u_{i} \text {, for any } x \in M\right\} .
$$

When $\partial \mathrm{F}\left(\chi^{0}\right) \neq \varnothing$, we say the fuzzy mapping $\mathrm{F}$ is sub-differentiable at $x^{0}$.

Obviously sub-differential ensures local sub-differential, the converse is not necessarily true. So in the last section Theorems 3.4, 3.6, and 3.7 about local sub-differential are gained. But there is different conclusion for Theorem 3.8, as follows:

Theorem 4.2. Let $\mathrm{F}: M \rightarrow \mathcal{F}_{0}$ be a fuzzy mapping, then $\partial \mathrm{F}(\mathrm{x})$ is closed convex set or empty set.

Proof. Let $\partial \mathrm{F}(\mathrm{x}) \neq \varnothing$, then $\partial \mathrm{F}(\mathrm{x})$ the proof is similar to the proof of Theorem 3.8, so we omit it.

We will prove $\partial F(x)$ is closed set.

Let $u^{m}=\left(u_{1}^{m}, u_{2}^{m}, \cdots, u_{n}^{m}\right) \in \partial F(x)(m=1,2, \cdots)$ and $\lim _{m \rightarrow \infty} D_{n}\left(u^{m}, u\right)=0$, then by Theorem 2.3, we have

$$
\begin{aligned}
\lim _{m \rightarrow \infty} D_{n}\left(u^{m}, u\right)=\left(\sum_{i=1}^{n}\left(D\left(u_{i}^{m}, u_{i}\right)\right)^{p}\right)^{\frac{1}{p}}=0(p \geqslant 1) \\
\quad \Longleftrightarrow \lim _{m \rightarrow \infty} D\left(u_{i}^{m}, u_{i}\right)=\lim _{m \rightarrow \infty} \sup _{r \in[0,1]} \max \left\{\left|u_{i *}^{m}(r)-u_{i *}(r)\right|,\left|u_{i}^{m *}(r)-u_{i}^{*}(r)\right|\right\}=0(i=1,2, \cdots, n) \\
\quad \Longleftrightarrow \lim _{m \rightarrow+\infty}\left|u_{i *}^{m}(r)-u_{i *}(r)\right|=0 \text { and } \lim _{m \rightarrow+\infty}\left|u_{i}^{m *}(r)-u_{i}^{*}(r)\right|=0(i=1,2, \cdots, n) \text { for any } r \in[0,1], \\
\quad \Longleftrightarrow \lim _{m \rightarrow+\infty} u_{i *}^{m}(r)=u_{i *}(r) \text { and } \lim _{m \rightarrow+\infty} u_{i}^{m *}(r)=u_{i}^{*}(r)(i=1,2, \cdots, n) \text { for any } r \in[0,1] .
\end{aligned}
$$

On the other hand, form $u_{m} \in \partial F(x)(m=1,2, \cdots)$, we have

$$
F(y)+\sum_{y_{i}<x_{i}}\left|y_{i}-x_{i}\right| u_{i}^{m}(r) \geqslant F(x)+\sum_{y_{i} \geqslant x_{i}}\left(y_{i}-x_{i}\right) u_{i}^{m}(r)
$$




$$
\begin{aligned}
\Longleftrightarrow & F(y)_{*}(r)+\sum_{y_{i}<x_{i}}\left|y_{i}-x_{i}\right| u_{i *}^{m}(r) \geqslant F(x)_{*}(r)+\sum_{y_{i} \geqslant x_{i}}\left(y_{i}-x_{i}\right) u_{i *}^{m}(r), \\
& F(y)^{*}(r)+\sum_{y_{i}<x_{i}}\left|y_{i}-x_{i}\right| u_{i}^{m *}(r) \geqslant F(x)^{*}(r)+\sum_{y_{i} \geqslant x_{i}}\left(y_{i}-x_{i}\right) u_{i}^{m *}(r),
\end{aligned}
$$

for any $y \in M$.

Therefore, let $m \rightarrow \infty$, then by (4.1) and (4.2), we have

$$
F(y)_{*}(r)+\sum_{y_{i}<x_{i}}\left|y_{i}-x_{i}\right| u_{i *}(r) \geqslant F(x)_{*}(r)+\sum_{y_{i} \geqslant x_{i}}\left(y_{i}-x_{i}\right) u_{i *}(r)
$$

for any $y \in M$, i.e., $u=\left(u_{1}, u_{2}, \cdots, u_{n}\right) \in \partial F(x)$. So $\partial F(x)$ is closed set.

\section{Convex extensibility of fuzzy mapping}

By means of the convexification method (Definition 2.4), we study the relations between the subdifferential of fuzzy mappings and the sub-differential of convexification fuzzy mappings, then we will obtain the sufficiency conditions of existence of convex extension for fuzzy mappings.

Theorem 5.1. Let $\mathrm{F}_{\mathrm{c}}$ be the convexification fuzzy mapping of $\mathrm{F}: \mathrm{M} \rightarrow \mathcal{F}_{0}, x^{0} \in \mathrm{M}$, then $\mathrm{F}$ is sub-differentiable at $x^{0}$ if and only if $\mathrm{F}_{\mathrm{c}}\left(\mathrm{x}^{0}\right)=\mathrm{F}\left(\mathrm{x}^{0}\right)$ and $\mathrm{F}_{\mathrm{c}}$ is also sub-differentiable at $x^{0}$.

Proof. Necessity: Let $F$ be sub-differentiable at $x^{0}$, then there exists

$$
u=\left(u_{1}, u_{2}, \ldots, u_{n}\right) \in \mathcal{F}_{0}^{n}
$$

such that

$$
F(x)+\sum_{x_{i}<x_{i}^{0}}\left|x_{i}-x_{i}^{0}\right| u_{i} \geqslant F\left(x^{0}\right)+\sum_{x_{i} \geqslant x_{i}^{0}}\left(x_{i}-x_{i}^{0}\right) u_{i}
$$

for any $x \in M$. by

So, for any $m \in N, \alpha_{j}>0(j=1,2, \ldots, m), \sum_{j=1}^{m} \alpha_{j}=1, x^{j} \in M(j=1,2, \cdots, m)$ and $\sum_{j=1}^{m} \alpha_{j} x^{j}=x^{0}$,

$$
F\left(x^{j}\right)+\sum_{x_{i}^{j}<x_{i}^{0}}\left|x_{i}^{j}-x_{i}^{0}\right| u_{i} \geqslant F\left(x^{0}\right)+\sum_{x_{i}^{j} \geqslant x_{i}^{0}}\left(x_{i}^{j}-x_{i}^{0}\right) u_{i},
$$

we have

$$
\sum_{j=1}^{m} \alpha_{j} F\left(x^{j}\right)+\sum m_{j=1}^{m} \alpha_{j}\left(\sum_{x_{i}^{j}<x_{i}^{0}}\left(x_{i}^{0}-x_{i}^{j}\right)\right) u_{i} \geqslant F\left(x^{0}\right)+\sum_{j=1}^{m} \alpha_{j}\left(\sum_{x_{i}^{j} \geqslant x_{i}^{0}}\left(x_{i}^{j}-x_{i}^{0}\right)\right) u_{i},
$$

i.e.,

$$
\sum_{j=1}^{m} \alpha_{j} F\left(x^{j}\right)+\sum_{x_{i}^{j}<x_{i}^{0}}\left(x_{i}^{0}-\sum m_{j=1}^{m} \alpha_{j} x_{i}^{j}\right) u_{i} \geqslant F\left(x^{0}\right)+\sum_{x_{i}^{j} \geqslant x_{i}^{0}}\left(\sum_{j=1}^{m} \alpha_{j} x_{i}^{j}-x_{i}^{0}\right) u_{i} .
$$

On the other hand, for $x^{0}=\left(x_{1}^{0}, x_{2}^{0}, \ldots, x_{n}^{0}\right), x^{j}=\left(x_{1}^{j}, x_{2}^{j}, \ldots, x_{n}^{j}\right)(j=1,2, \ldots, m)$, we know

$$
\sum m_{j=1}^{m} \alpha_{j} x^{i}=x^{0} \Longleftrightarrow \sum m_{j=1} \alpha_{j} x_{i}^{j}=x_{i}^{0}(i=1,2, \ldots, n) .
$$

Therefore, $\sum_{j=1}^{m} \alpha_{j} F\left(x^{i}\right) \geqslant F\left(x^{0}\right)$, thus we obtain

$$
\begin{aligned}
F_{C}\left(x^{0}\right) & =\inf _{m \in N}\left\{\sum_{j=1}^{m} \alpha_{j} F\left(x^{j}\right) \mid \alpha_{j}>0, x^{j} \in M(j=1,2, \cdots, m), \sum_{j=1}^{m} \alpha_{j}=1, \sum_{j=1}^{m} \alpha_{j} x^{j}=x_{0}\right\} \\
& \geqslant F\left(x^{0}\right) .
\end{aligned}
$$


By Theorem 2.5, we easily gain $F_{c}\left(x^{0}\right) \leqslant F\left(x^{0}\right)$. So $F\left(x^{0}\right)=F\left(x^{0}\right)$.

Now, we will prove that $F_{c}$ is sub-differentiable at $x^{0}$.

For $x \in \operatorname{CoM}$, there exist $\alpha_{j}>0(j=1,2, \cdots, m), \sum_{j=1}^{m} \alpha_{j}=1$, and $x^{j} \in M(j=1,2, \cdots, m)$, such that $\sum_{j=1}^{m} \alpha_{j} x^{j}=x$.

By $F$ is sub-differentiable at $x^{0}$, there exists $u=\left(u_{1}, u_{2}, \cdots, u_{n}\right) \in \mathcal{F}_{0}^{n}$ such that

$$
F\left(x^{j}\right)+\sum_{x_{i}^{j}<x_{i}^{0}}\left|x_{i}^{j}-x_{i}^{0}\right| u_{i} \geqslant F(x)+\sum_{x_{i}^{j} \geqslant x_{i}^{0}}\left(x_{i}^{j}-x_{i}\right) u_{i},(j=1,2, \cdots, m) \text { for any } x \in M .
$$

So

$$
\sum m_{j=1} \alpha_{j} F\left(x^{j}\right)+\sum_{x_{i}^{j}<x_{i}^{0}} \sum_{j=1}^{m}\left(x_{i}^{0}-x_{i}^{j}\right) u_{i} \geqslant F\left(x_{0}\right)+\sum_{x_{i}^{j} \geqslant x_{i}^{0}} \sum_{j=1}^{m}\left(x_{i}^{j}-x_{i}^{0}\right) u_{i} .
$$

It follows from the fact $x_{i}^{j}<x_{i}^{0}$ implies $\sum_{j=1}^{m} \alpha_{j} x_{i}^{j}=x_{i}<x_{i}^{0}$, that

$$
\sum_{j=1}^{m} \alpha_{j} F\left(x^{j}\right)+\sum_{x_{i}^{j}<x_{i}^{0}}\left(x_{i}^{0}-x_{i}\right) u_{i} \geqslant F\left(x_{0}\right)+\sum_{x_{i}^{j} \geqslant x_{i}^{0}}\left(x_{i}-x_{i}^{0}\right) u_{i} .
$$

Therefore, we have

$$
\begin{aligned}
& \inf _{m \in N}\left\{\sum_{j=1}^{m} \alpha_{j} F\left(x^{j}\right) \mid \alpha_{j}>0, x^{j} \in M(j=1,2, \cdots, m), \sum_{j=1}^{m} \alpha_{j} x^{j}=x^{0}\right\} \\
& \quad+\sum_{x_{i}<x_{i}^{0}}\left(x_{i}^{0}-x_{i}\right) u_{i} \geqslant F\left(x^{0}\right)+\sum_{x_{i} \geqslant x_{i}^{0}}\left(x_{i}-x_{i}^{0}\right) u_{i} .
\end{aligned}
$$

By $F_{C}\left(x^{0}\right)=F\left(\chi^{0}\right)$, we have

$$
F_{C}\left(x^{0}\right)+\sum_{x_{i}<x_{i}^{0}}\left(x_{i}^{0}-x_{i}\right) u_{i} \geqslant F_{C}\left(x^{0}\right)+\sum_{x_{i} \geqslant x_{i}^{0}}\left(x_{i}-x_{i}^{0}\right) u_{i} .
$$

So $u=\left(u_{1}, u_{2}, \cdots, u_{n}\right) \in \partial F\left(\chi^{0}\right)$, that is, $F_{C}$ is sub-differentiable at $\chi^{0}$.

Sufficiency: since $F_{c}$ is sub-differentiable at $x^{0}$, there exists $u \in \mathcal{F}_{0}^{n}$, such that

$$
F_{C}(x)+\sum_{x_{i}<x_{i}^{0}}\left(x_{i}^{0}-x_{i}\right) u_{i} \geqslant F\left(x^{0}\right)+\sum_{x_{i} \geqslant x_{i}^{0}}\left(x_{i}-x_{i}^{0}\right) u_{i} \text { for any } x \in \operatorname{CoM} .
$$

It follows from $\mathrm{F}_{\mathrm{C}}\left(x^{0}\right)=\mathrm{F}\left(x^{0}\right)$ and $M \subset \mathrm{CoM}$, that

$$
F(x)+\sum_{x_{i}<x_{i}^{0}}\left(x_{i}^{0}-x_{i}\right) u_{i} \geqslant F_{c}(x)+\sum_{x_{i}<x_{i}^{0}}\left(x_{i}^{0}-x_{i}\right) u_{i} \geqslant F_{c}\left(x^{0}\right)+\sum_{x_{i} \geqslant x_{i}^{0}}\left(x_{i}-x_{i}^{0}\right) u_{i},
$$

for any $x \in \operatorname{CoM}$. Thus we have $u \in \partial F\left(x^{0}\right)$, that is, $F$ is sub-differentiable at $x$.

Theorem 5.2. Let $\mathrm{F}: M \rightarrow \mathcal{F}_{0}$ be a lower semi-continuous fuzzy mapping. If for any convex extreme subset $\mathrm{E}(\operatorname{dim} \mathrm{E}>0)$ of $\mathrm{CoM}$, the set $\mathrm{B}$ where the sub-differential of $\left.\mathrm{F}\right|_{\mathrm{E} \cap \mathrm{D}}$ exists is dense in $\mathrm{E} \cap \mathrm{M}$, then

(1) The convexification fuzzy mapping $F_{c}$ of $F$ is the extension of $F$.

(2) The sub-differential of $\left.\mathrm{F}_{\mathrm{C}}\right|_{\mathrm{E}}$ is on $\mathrm{B}$. 
Proof. By lower semi-continuity of $F$, we can easily prove $F$ is a lower bound. Therefore, by Theorems 2.2 and Theorems 2.5 , we can easily prove the convexification fuzzy mapping $F_{c}$ of $F$ must exist.

(1) Let $x^{0} \in M$. If $x^{0}$ is an extreme point of $M$, then have $F_{c}\left(x^{0}\right)=F\left(x^{0}\right)$.

If $x^{0}$ is not an extreme point of $M$, then there exist $x^{j} \in M, \lambda_{j}>0(j=1,2, \cdots, m)$ and $\sum_{j=1}^{m} \lambda_{j}=1$, such that

$$
\sum_{j=1}^{m} \lambda_{j} x^{j}=x^{0}
$$

Let $E$ be the minimum convex extreme subset of $\operatorname{CoM}$ containing $x^{0}, x^{1}, \ldots, x^{m}$. Since $x_{0}$ is not an extreme point, so dimE $>0$.

Next, we will prove $\operatorname{Co}(E \cap M)=E$.

By $E \cap M \subset E$, we have

$$
\operatorname{Co}(E \cap M) \subset \operatorname{CoE}=E \text {. }
$$

Conversely, for $y \in E \subset C o M$, there exist $y^{j} \in M, \beta_{j}>0(j=1,2, \cdots, m)$ and $\sum_{j=1}^{m} \beta_{j}=1$, such that $\sum_{j=1}^{m} \beta_{j} y^{j}=y$.

Since $E$ is the extreme subset, $y^{j} \in E(j=1,2, \cdots, m)$, hence

$$
y^{j} \in E \cap M(j=1,2, \cdots, m) \text {. }
$$

Therefore

$$
y \in \operatorname{Co}(E \cap M)
$$

So $\operatorname{Co}(E \cap M)=E$.

Next, we prove that $x^{0} \in i c r E$ (where icrE is the relative interior point set of $E$ ).

Assume $x^{0} \notin i c r E$, then $x^{0} \in E / i c r E$, by the separation theorem of convex sets, there exists

$$
x^{*} \in R^{n}
$$

such that

$$
<x^{*}, x^{0}>\geqslant \sup _{y \in E}<x^{*}, y>
$$

Hence

$$
<x^{*}, x^{j}>\leqslant<x^{*}, x^{0}>(j=1,2, \cdots, m) .
$$

It follows from

$$
<x^{*}, \sum_{j=1}^{m} \lambda_{j} x^{j}>=<x^{*}, x^{0}>
$$

that

$$
<x^{*}, x_{j}>=<x^{*}, x^{0}>(j=1,2, \cdots, m) .
$$

Let

$$
A=\left\{y \in E \mid<x^{*}, y>=<x^{*}, x^{0}>\right\},
$$

then we easily prove $A \subset E$ and $A$ is the convex extreme subset of $E$. So $A$ is the convex extreme subset of CoM and containing $x^{0}, x^{1}, \ldots, x^{m}$.

This contradicts $E$ is the minimum convex extreme subset of CoM containing $x^{0}, x^{1}, \ldots, x^{m}$. Thus $\chi^{0} \in$ icrE.

Let $G: E \rightarrow \mathcal{F}_{0}(\mathrm{Co}(E \cap M)=E)$ be the convexification fuzzy mapping of $\left.F\right|_{E \cap M}$, then for any $r \in[0,1]$, $\mathrm{G}(\mathrm{x})_{*}(\mathrm{r})$ and $\mathrm{G}(\mathrm{x})^{*}(\mathrm{r})$ are the real-valued convex functions on $\mathrm{E}$. Hence it is continuous at $x_{0} \in \mathrm{icr} E$.

Since the set $B$ where the sub-differential of $\left.F\right|_{E \cap M}$ exists is dense in $E \cap M$. So, for $x^{0} \in E \cap M$, exists is $\left\{z^{\mathrm{m}}\right\} \subset \mathrm{B}$ such that $z^{\mathrm{m}} \rightarrow x^{0}(\mathrm{~m} \rightarrow \infty)$. By Theorem 5.1, we have

$$
\mathrm{F}\left(z^{\mathrm{m}}\right)=\mathrm{G}\left(z^{\mathrm{m}}\right)=\left.\mathrm{F}\right|_{\mathrm{E} \cap M}\left(z^{\mathrm{m}}\right) .
$$


Because $F$ is lower semi-continuous, $F(x)_{*}(r)$ and $F(x)^{*}(r)$ are lower semi-continuous at $x^{0} \in$ icrE (by Theorems 2.8). By Remark 2.9, for any $r \in[0, r]$, we have

$$
\begin{aligned}
& F\left(x^{0}\right)_{*}(r) \leqslant \varliminf_{n \rightarrow \infty} F\left(z^{m}\right)_{*}(r)=\lim _{n \rightarrow \infty} G\left(z^{m}\right)_{*}(r)=G\left(x^{0}\right)_{*}(r), \\
& F\left(x^{0}\right)^{*}(r) \leqslant \varliminf_{n \rightarrow \infty} F\left(z^{m}\right)^{*}(r)=\lim _{n \rightarrow \infty} G\left(z^{m}\right)^{*}(r)=G\left(x^{0}\right)^{*}(r) .
\end{aligned}
$$

Thus we can obtain

$$
F\left(x^{0}\right) \leqslant G\left(x^{0}\right)
$$

So

$$
G\left(x^{0}\right)=\inf _{m \in N}\left\{\sum_{j=1}^{m} \lambda_{j} F\left(x^{j}\right) \mid \lambda_{j}>0, x^{j} \in M \cap E(j=1,2, \cdots, m), \sum_{j=1}^{m} \lambda_{j}=1, \sum_{j=1}^{m} \lambda_{j} x^{j}=x^{0}\right\} \leqslant \sum_{j=1}^{m} \lambda_{j} F\left(x^{j}\right) .
$$

Therefore, we have $F\left(x^{0}\right) \leqslant \sum_{j=1}^{m} \lambda_{j} F\left(x^{j}\right)$, thus

$$
F\left(x^{0}\right) \leqslant \inf _{m \in N}\left\{\sum_{j=1}^{m} \lambda_{j} F\left(x^{j}\right) \mid \lambda_{j}>0, x^{j} \in M(i=1,2, \cdots, m), \sum_{j=1}^{m} \lambda_{j}=1, \sum_{j=1}^{m} \lambda_{j} x^{j}=x^{0}\right\},
$$

i.e., $F\left(x^{0}\right) \leqslant F_{C}\left(x^{0}\right)$.

On the other hand, $F\left(x^{0}\right) \geqslant F_{C}\left(x^{0}\right)$. So $F\left(x^{0}\right)=F_{C}\left(x^{0}\right)$. Thus, by $M \subset C o M$ we have $F$ is the extension of $F$.

(2) We now prove that the sub-differential of $\left.F_{C}\right|_{E}$ exists on $B$.

For any $x \in B$, by the known conditions, we have $\left.\partial F\right|_{E \cap D}(x) \neq \varnothing$. Next, we prove

$$
\left.\left.\partial \mathrm{F}_{\mathrm{C}}\right|_{\mathrm{E}}(\mathrm{x}) \supset \partial \mathrm{F}\right|_{\mathrm{E} \cap M}(\mathrm{x}) \text {. }
$$

For $y \in E \subseteq$ CoM, take $y^{j} \in M, \lambda_{j}>0(j=1,2, \cdots, m)$, and $\sum_{j=1}^{m} \lambda_{j}=1$, such that $y=\sum_{j=1}^{m} \lambda_{j} y^{j}$. Since $E$ is the extreme subset and $y^{j} \in E(j=1,2, \cdots, m)$, we have

$$
y_{j} \in M \cap E(j=1,2, \cdots, m) \text {. }
$$

Hence, for $u^{x}=\left.\left(u_{1}^{x}, u_{2}^{x}, \ldots, u_{n}^{x}\right) \in \partial F\right|_{E \cap M}(x)$, we have

$$
F\left(y^{j}\right)+\sum_{y_{i}^{j}<x_{i}}\left(x_{i}-y_{i}^{j}\right) u_{i}^{x} \geqslant F(x)+\sum_{y_{i}^{j} \geqslant x_{i}}\left(y_{i}^{j}-x_{i}\right) u_{i}^{x}(j=1,2, \ldots, m) .
$$

So

$$
\sum_{j=1}^{m} \lambda_{j} F\left(y^{j}\right)+\sum_{y_{i}^{j}<x_{i}}\left(x_{i}-\sum_{j=1}^{m} \lambda_{j} y_{i}^{j}\right) u_{i}^{x} \geqslant F(x)+\sum_{y_{i}^{j}<x_{i}}\left(\sum_{j=1}^{m} \lambda_{j} y_{i}^{j}-x_{i}\right) u_{i}^{x},
$$

i.e.,

$$
\sum_{j=1}^{m} \lambda_{j} F\left(y^{j}\right)+\sum_{y_{i}^{j}<x_{i}}\left(x_{i}-y_{i}^{j}\right) u_{i}^{x} \geqslant F(x)+\sum_{y_{i}^{j}<x_{i}}\left(y_{i}^{j}-x_{i}\right) u_{i}^{x} .
$$

Therefore,

$$
\inf _{m \in N}\left\{\sum_{j=1}^{m} \lambda_{j} F\left(y^{j}\right) \mid \lambda_{j}>0, y^{j} \in M(j=1,2, \cdots, m), \sum^{m} \lambda j=1, \sum_{j=1}^{m} \lambda_{j} y^{j}=y\right\}
$$




$$
+\sum_{x_{i}<y_{i}}\left(x_{i}-y_{i}\right) u_{i}^{x} \geqslant F(x)+\sum_{y_{i} \geqslant x_{i}}\left(y_{i}-x_{i}\right) u_{i}^{x} .
$$

Because $F_{C}(x)=F(x)$ on $B \subset M$. Hence, for any $y \in E$, there has

$$
F_{C}(y)+\sum_{x_{i}<y_{i}}\left(x_{i}-y_{i}\right) u_{i}^{x} \geqslant F(x)+\sum_{y_{i} \geqslant x_{i}}\left(y_{i}-x_{i}\right) u_{i}^{x} .
$$

Thus, $\left.\mathrm{u}^{\mathrm{x}} \in \partial \mathrm{F}\right|_{\mathrm{E}}(\mathrm{x})$, so $\left.\left.\partial \mathrm{F}\right|_{\mathrm{E}}(\mathrm{x}) \supset \partial \mathrm{F}\right|_{\mathrm{E} \cap \mathrm{D}}(\mathrm{x})$. By the arbitrariness of $x$, the sub-differential of $\left.\mathrm{F}_{C}\right|_{\mathrm{E}}$ exists on B.

By Theorem 5.2, we easily obtain the following Corollary 5.3.

Corollary 5.3. Let $\mathrm{F}: M \rightarrow \mathcal{F}_{0}$ be a lower semi-continuous fuzzy mapping and $M$ be a convex set. If the set where the sub-differential is of $\left.\mathrm{F}\right|_{\mathrm{E}}$ is dense in $\mathrm{E}$ for any convex extreme subset $\mathrm{E}$ of $\mathrm{M}$, then $\mathrm{F}$ is convex fuzzy mapping.

\section{Conclusion}

In [14], the concept of sub-differential of fuzzy mapping is introduced by Wang and Wu. In this paper, we call it the local sub-differential, then we study the basic properties of local sub-differential, obtain some results which are similar to general sub-differential. These conclusions are helpful to study the approximation and regulation of sub-differential of fuzzy mapping and related issues. It is well-known that not every fuzzy mapping can be extended to convex fuzzy mapping. Hence convex extension of general fuzzy mapping is a natural and important problem which is helpful to improve the efficiency of the global optimization method. In this paper, in order to study the convex extension of fuzzy mapping, we generalize the concept of local sub-differential of fuzzy mapping, which has the basic properties of local sub-differential. And its sub-differential is an empty set or closed convex set. As an application, based on the new sub-differential of fuzzy mapping and convex method of fuzzy mapping, we study the relations between sub-differential of general fuzzy mapping and sub-differential of its convex fuzzy mapping, obtain a sufficient condition that lower semi-continuous fuzzy mapping can be extended to convex fuzzy mapping. According to these conclusions, we propose a new method to research optimization problems of general fuzzy mapping.

\section{Acknowledgment}

This work is supported by the National Science Fund of China (11461052) and the Inner Mongolia Natural Science Foundation of China(2014MS0107).

\section{References}

[1] E. Ammar, J. Metz, On fuzzy convexity and parametric fuzzy optimization, Fuzzy Sets and Systems, 49 (1992), $135-141$. 1

[2] Y. E. Bao, C.-X. Wu, Convexity and strict convexity of fuzzy mappings, (Chinese) J. Harbin Inst. Tech., 39 (2007), 639-641.

[3] Y. E. Bao, C.-X. Wu, Semistrictly convex fuzzy mappings, J. Math. Res. Exposition, 30 (2010), 571-580. 1

[4] B. Bede, S. G. Gal, Generalizations of the differentiability of fuzzy-number-valued functions with applications to fuzzy differential equations, Fuzzy Sets and Systems, 151 (2005), 581-599. 1

[5] B. Bede, L. Stefanini, Generalized differentiability of fuzzy-valued functions, Fuzzy Sets and Systems, 230 (2013), 119141. 1

[6] S. S. L. Chang, L. A. Zadeh, On fuzzy mapping and control, IEEE Trans. Systems, Man, and Cybernet., SMC-2 (1972), 30-34. 1

[7] D. Dubois, H. Prade, Towards fuzzy differential calculus, I, II, III, Fuzzy Sets and Systems, 8 (1982), 1-17, 105-116, 225-234. 1

[8] Z. T. Gong, H. X. Li, Derivatives and gradients of fuzzy mappings and their applications, (Chinese) Appl. Math. J. Chinese Univ. Ser. A, 25 (2010), 229-238. 1 
[9] S. Nanda, K. Kar, Convex fuzzy mappings, Fuzzy Sets and Systems, 48 (1992), 129-132. 1

[10] M. Panigrahi, G. Panda, S. Nanda, Convex fuzzy mapping with differentiability and its application in fuzzy optimization, European J. Oper. Res., 185 (2008), 47-62. 1, 3.2

[11] M. L. Puri, D. A. Ralescu, Differentials of fuzzy functions, J. Math. Anal. Appl., 91 (1983), 552-558. 1

[12] R. T. Rockafellar, Convex analysis, Princeton Mathematical Series, Princeton University Press, Princeton, N.J., (1970). 2.9

[13] Y.-R. Syau, Differentiability and convexity of fuzzy mappings, Comput. Math. Appl., 41 (2001), 73-81. 1

[14] G.-X. Wang, C.-X. Wu, Directional derivatives and subdifferential of convex fuzzy mappings and application in convex fuzzy programming, Fuzzy Sets and Systems, 138 (2003), 559-591. 1, 2, 2.1, 2.6, 6

[15] C. X. Wu, C. Wu, The supremum and infimum of the [a] set of fuzzy numbers and its [their] application, J. Math. Anal. Appl., 210 (1997), 499-511. 2, 2.2, 2.7

[16] C. Zhang, X.-H. Yuan, E. S. Lee, Duality theory in fuzzy mathematical programming problems with fuzzy coefficients, Comput. Math. Appl., 49 (2005), 1709-1730. 1

[17] C. Zhang, X.-H. Yuan, E. S. Lee, Convex fuzzy mapping and operations of convex fuzzy mappings, Comput. Math. Appl., 51 (2006), 143-152. 1, 2.4, 2.5 